\title{
INFLUÊNCIA DE GENÓTIPOS DE COUVE (Brassica oleracea L.var.acephala DC.) NA BIOLOGIA DE Plutella xylostella (L., 1758) (Lepidoptera: Plutellidae)
}

\author{
Influence of cabbage genotypes (Brassica oleracea L. var. acephala DC.) on \\ the biology of Plutella xylostella (L., 1758) (Lepidoptera: Plutellidae) \\ Arlindo Leal Boiça Junior'1, Sônia Regina Alves Tagliari², Rafael Major Pitta ${ }^{3}$, \\ Flávio Gonçalves de Jesus ${ }^{4}$, Leila Trevizan $\mathrm{Braz}^{5}$
}

\begin{abstract}
RESUMO
Objetivou-se, com este trabalho, avaliar a influência de alguns genótipos de couve (Brassica oleracea L. var. acephala DC.) no desenvolvimento de Plutella xylostella (L., 1758) (Lepidoptera: Plutellidae). Os genótipos avaliados foram: Manteiga de Ribeirão Pires I-2620, Roxa I-919, Manteiga de São José, Manteiga de Monte Alegre, Pires 2 de Campinas, Couve Comum, Couve de Arthur Nogueira 2, Couve de Arthur Nogueira 1. Lagartas recém-eclodidas foram mantida em discos foliares de $8 \mathrm{~cm}$ de diâmetro para cada genótipo. Foram analisados os seguintes parâmetros: duração e viabilidade das fases larval e pupal, longevidade e fecundidade de adultos, utilizando análises paramétricas e de agrupamentos para interpretação dos dados. Observou-se um prolongamento em dias no ciclo de $P$. xylostella, aumento no peso de pupa e maiores valores de viabilidade e fecundidade, durante a segunda geração. O genótipo Couve de Arthur Nogueira 2 foi menos favorável ao desenvolvimento de $P$. xylostella nas duas gerações, e Couve Comum demonstrou maior influência negativa ao inseto na segunda geração. Manteiga de Ribeirão Pires I-2620 foi o mais suscetível nas duas gerações, agrupando com este na segunda geração Pires 2 de Campinas e Manteiga de São José.
\end{abstract}

Termo para indexação: Brassicaceae, traça-das-cruciferas, resistência de plantas, antibiose.

\section{ABSTRACT}

The purpose of this study was to evaluate the effect of cabbage genotypes (Brassica oleracea L. var. acephala DC.) on growth of Plutella xylostella (Lepidoptera: Plutellidae). The genotypes evaluated were: Manteiga of Ribeirão Pires I-2620, Roxa I919, Manteiga of São José, Manteiga of Monte Alegre, Pires 2 of Campinas, Couve Comum, Couve of Arthur Nogueira 2, Couve of Arthur Nogueira 1. Neonate larvae were reared in $8 \mathrm{~cm}$ leaf discs of each genotype. The parameters evaluated were: period and viability of the larval and pupal stages, sex ratio, longevity and fecundity of adults. Parametric and Cluster analyses were used for data analysis. Overall, it was observed a developmental delay in the $P$. xylostella cicle, larger pupal, higher viability and fecundity during the second generation. Couve of Arthur Nogueira 2 was less favorable to the development of $P$. xylostella in the two generations, and Couve Comum was not an appropriate host for the insect in the second generation. Manteiga of Ribeirão Pires I-2620 was the most susceptible in both generations belonging to the same cluster with Pires 2 of Campinas and Manteiga of São José in the second generation.

Index terms: Brassicaceae, diamondback moth, plant resistance, antibiosis.

(Recebido em 27 de fevereiro de 2009 e aprovado em 29 de julho de 2010)

\section{INTRODUÇÃO}

A traça-das-crucíferas, Plutella xylostella (LINNEAUS, 1758) (Lepidoptera: Plutellidae), é um microlepidóptero cosmopolita com ampla distribuição em regiões produtoras de brássicas no mundo (MARANHÃO et al., 1998; GALLO et al., 2002; SARFRAZ et al., 2006; BACCI et al., 2009). Trata-se de uma praga considerada limitante as crucíferas em áreas tropicais, em razão dos danos provocados, seu ciclo curto e alto potencial reprodutivo, promovendo elevado número de gerações por ano (ULMER et al., 2002).

Esse comportamento cosmopolita exibido por $P$. xyllostella proporciona fácil encontro de hospedeiros para seu desenvolvimento durante todo o ano. Além disso, no início da fase larval, encontra-se, na maioria das vezes, protegida no interior da folha do hospedeiro, dificultando o seu controle (NOGUEIRA, 1981).

\footnotetext{
'Universidade Estadual Paulista "Júlio de Mesquita Filho"/UNESP - Faculdade de Ciências Agrárias e Veterinárias/FCAV - Departamento de Fitossanidade Professor Paulo Donato Castellane s/n - 14884-900 - Jaboticabal, SP - aboicajr@fcav.unesp.br

"Universidade Estadual Paulista "Júlio de Mesquita Filho"/UNESP - Faculdade de Ciências Agrárias e Veterinárias/FCAV - Jaboticabal, SP ${ }^{3}$ Embrapa Agrossilvipastoril - Sinop, MT

${ }^{4}$ Instituto Federal Goiano - Urutaí, GO

${ }^{5}$ Universidade Estadual Paulista “Júlio de Mesquita Filho"/UNESP - Faculdade de Ciências Agrárias e Veterinárias/FCAV - Departamento de Produção Vegetal - Jaboticabal, SP
} 
No Brasil, alguns resultados foram relatados quanto à resistência de diferentes cultivares de brássicas à traça-dascrucíferas, esses resultados são baseados principalmente em dados biológicos do desenvolvimento da praga, atratividade para alimentação e oviposição em relação ao hospedeiro e característica da planta hospedeira (BARROS; VENDRAMIM, 1999; TORRES et al., 2006; THULER et al., 2007; BOIÇA JUNIOR; CHAGAS FILHO, 2009).

A resistência de plantas à $P$. xylostella tem sido avaliada com base em duas características morfológica: a cerosidade da superfície foliar, determinada pelo teor de alcano, e o teor de sinigrina presente nas folhas (EIGENBRODE et al., 1991; SPENCER et al., 1999; ULMER et al., 2002).

Thuler et al. (2007), avaliando os graus de resistência em diferentes variedades de brássicas à $P$. xyllostela, observam que Chato-de-quintal foi moderadamente resistente à praga. Boiça Junior e Chagas Filho (2009) visualizaram menores consumos no híbrido Silver Streak.

Como a cultura é principalmente utilizada por pequenos produtores, o uso de variedades resistentes, ou a associação com outro método de controle, representa uma alternativa que poderá não apenas reduzir as populações das pragas, mas também viabilizar sua produção, uma vez que diminui a quantidade de inseticidas e resíduos (BOIÇA JUNIOR; CHAGAS FILHO, 2009).

Objetivou-se, com este trabalho, avaliar a influência de alguns genótipos de couve (Brassica oleracea L. var. acephala DC.) no desenvolvimento de P. xylostella.

\section{MATERIAL E MÉTODOS}

Os experimentos foram conduzidos no Laboratório de Resistência de Plantas a Insetos do Departamento de Fitossanidade na Faculdade de Ciências Agrárias e Veterinárias (FCAV) - Campus de Jaboticabal, no período de fevereiro a junho de 2007. As condições de laboratório para a criação e condução dos experimentos foram: temperatura de $25 \pm 2^{\circ} \mathrm{C}$, umidade relativa $70 \pm 10 \%$ e fotofase de 12 horas.

Para a realização dos experimentos, foi mantida uma criação estoque de $P$. xylostella, utilizando-se insetos coletados inicialmente em culturas de crucíferas na região de Jaboticabal. Larvas e adultos de P. xyllostella foram criados de acordo com a metodologia descrita em Barros e Vendramim (1999), Torres et al. (2006) e Boiça Junior e Chagas Filho (2009).

$\mathrm{Na}$ condução dos experimentos, foram utilizadas folhas dos genótipos de couve: Manteiga de Ribeirão Pires I-2620, Roxa I-919, Manteiga de São José, Manteiga de
Monte Alegre, Pires 2 de Campinas, Couve Comum, Couve de Arthur Nogueira 2, Couve de Arthur Nogueira 1, sendo utilizados discos foliares de $8 \mathrm{~cm}$ de diâmetro de cada genótipo, acondicionados em placas de Petri forrada com papel filtro de acordo com Torres et al. (2006), onde confinaram-se 12 lagartas de $P$. xylostella recém-eclodidas. Para cada um dos genótipos, utilizaram-se 10 placas equivalentes, constituindo-se as repetições do experimento. Esses genótipos utilizados, foram selecionados em testes de não-preferência para alimentação (TAGLIARI et al., 2006) e transplantados em outubro de 2006, cultivados em vasos com capacidade de $5 \mathrm{~kg}$.

Os discos foliares foram trocados a partir do quarto dia de confinamento, em decorrência do hábito minador das lagartas, após esse período foram feitas trocas diárias dos discos foliares de cada placa, realizadas até que as lagartas atingissem a fase de pupa. Esse procedimento repetiu-se para a segunda geração.

As lagartas, ao atingirem a fase de pupa, foram individualizadas em placas "ELISA" vedadas com filme plástico de "PVC", os quais foram perfurados para permitir a passagem de ar.

Para determinar a longevidade dos adultos, estes foram separados em grupos de 20 machos e 20 fêmeas em cada tratamento e, posteriormente, confinados nas placas "ELISA", cada inseto correspondeu a uma repetição. Para a avaliação do número de ovos por fêmea, outros 10 casais de cada tratamento foram separados por genótipo, contando-se diariamente, o número de ovos e, em seguida, colocados em placa de Petri até a eclosão das lagartas, para esse parâmetro adotou-se o total de 10 repetições.

No estudo da antibiose em relação à praga, avaliouse seguintes parâmetros biológicos: duração e viabilidade das fases larval e pupal (macho e fêmea), peso de pupa com 24 horas de idade, duração e viabilidade do período da eclosão da lagarta à emergência do adulto, razão sexual, número total de ovos por fêmea, número de ovos diários por fêmea e longevidade dos adultos.

Os resultados foram submetidos à análise de variância e as médias comparadas pelo teste de Tukey $(\mathrm{P}<0,05)$. Foi efetuado também a analise multivariada pelo método hierárquico de agrupamento em dendrograma, empregando o método de aglomeração de Ward e a distância de dissimilaridade euclidiana, para classificar as cultivares em grupos em relação à resistência a esse inseto.

\section{RESULTADOS E DISCUSSÃO}

Foram observadas diferenças significativas para os parâmetros biológicos viabilidade larval, período pupal de fêmea, viabilidade larval + pupal e longevidade de macho e 
fêmea para a primeira geração. Para a segunda geração, os parâmetros significativos foram período larval, viabilidade larval, período e viabilidade larval + pupal, peso pupal e longevidade de macho (Tabelas 1, 2 e 3).

Pelos dados, observa-se um prolongamento em dias no ciclo de $P$. xylostella, quando mantidas nos genótipos pela segunda geração, aumentando o período larval, pupal e longevidade dos adultos (Tabela 2). Segundo Lara (1991), o alongamento do ciclo aumenta a exposição da praga aos inimigos naturais e diminui o número de gerações no tempo, acarretando diminuição populacional do inseto.

$\mathrm{Na}$ viabilidade larval, os insetos alimentados nos genótipos Couve de Arthur Nogueira 1 e Couve de Arthur Nogueira 2 e Roxa I-919 apresentaram menores valores na segunda geração quando comparadas à primeira. $\mathrm{O}$ peso pupal apresentou maiores valores na segunda geração dos insetos alimentados em todos os genótipos, demonstrando adaptação ao hospedeiro (Tabelas 1 e 2).

Os dados referentes a viabilidade larval e pupal são semelhantes aos de Sarfraz et al. (2007) que avaliaram a biologia de $P$. xylostella em diversas espécies de brassicas, observando para couve viabilidades larval e pupal de 69 e $91,58 \%$.

As respostas observadas à menor viabilidade larval entre os genótipos, podem estar relacionadas a compostos químicos ativos, metabolizados pela planta, que se tornam toxinas fisiológicas e causando antibiose, ou substâncias deterrentes que suprimem a alimentação do inseto, reduzindo sua viabilidade, como sugerido por Eigenbrode et al. (1990).

Os resultados referentes à fecundidade (Tabela 3), também apresentaram maiores valores na segunda geração, evidenciando que a segunda geração proporcionaria um maior potencial reprodutivo por aumentar a viabilidade $\mathrm{e}$ fecundidade de P. xylostella.

A não diferenciação para a fecundidade pode ser atribuída às características das plantas, pois $P$. xylostella preferem ovipositar em folhas com as superfícies brilhantes quanto em comparação com as opacas (SHELTON; NAULT, 2004). Todos os genótipos estudados possuíam tal característica, oferecendo um estimulo visual para oviposição de maneira semelhante entre os materiais vegetais.

As médias das duas gerações foi superior às observadas por Torres et al. (2006), com fêmeas alimentadas na cultivar 'Portuguesa', porém observou-se que os parâmetros biológicos foram mais favoráveis ao desenvolvimento de $P$. xylostella na segunda geração, assim como neste estudo. Esses autores comentam que a praga pode estar se adaptando ao material vegetal do qual se alimenta, o que, consequentemente, pode vir manifestar suscetibilidade na próxima geração.

Pela análise de agrupamento (Figura 1), observa-se a separação de quatro grupos na primeira geração sendo 'Couve de Arthur Nogueira 2' (grupo 1), 'Manteiga de Monte Alegre' e 'Couve de Arthur Nogueira 1' (Grupo 2), 'Roxa I-919', 'Manteiga de São José', 'Pires 2 de Campinas', 'Comum' (grupo 3) e 'Manteiga de Ribeirão Pires I-2620' (grupo 4).

O genótipo Couve de Arthur Nogueira 2 (grupo 1) que apresentou os menores valores biológicos quanto ao desenvolvimento de $P$. xylostella (Tabela 1) não foi agrupado com nenhum genótipo. 'Manteiga de Ribeirão Pires I-2620' (Grupo 4) localizou-se ao oposto de 'Couve de Arthur Nogueira 2', também não agrupado com outros genótipos, e apresentando as melhores condições para o desenvolvimento do inseto.

Observa-se que os insetos criados em discos de folhas dos genótipos que agrupados após analise como grupo 1 apresentaram prolongamento do período larval, menor viabilidade larval, pupal e de larva + pupa, além de possuir o menor peso pupal, caracterizando que Couve de Arthur Nogueira 2 foi menos favorável ao desenvolvimento de $P$. xylostella, mesmo apresentando o ciclo de vida mais curto, característica essas de genótipos suscetíveis por suprir as necessidades nutricionais do inseto mais rapidamente. Entretanto, a porcentagem de indivíduos sobreviventes foi pequena, diminuindo a quantidade de descendentes para a próxima geração.

Inversamente, o grupo 4 demonstrou-se mais favorável ao desenvolvimento do inseto, contendo os maiores valores de viabilidade larval, pupal e larval + pupal. No entanto, proporcionou o ciclo mais longo, comportamento esse de genótipos resistentes, que prolongam o ciclo de vida dos insetos por proporcionarem menor ingestão de alimento em razão da presença de substâncias deterrentes ou por ocorrer desequilíbrio nutricional (HERNANDEZ; VENDRAMIM, 1997). No entanto, proporcionou pupas mais pesadas quando comparado com os outros grupos, e o segundo maior valor de fecundidade, evidenciando que os insetos estavam adaptando-se ao hospedeiro.

Dados semelhantes a estes de longevidade foram observados por Thuler et al. (2007) para o genótipos de couve 'Geórgia' e cultivares comerciais de repolho, embora não tenham diferido significativamente entre si, enquanto Gonçalves e Di Mare (2005), estudando a biologia de populações de $P$. xylostella endo e exocruzadas alimentadas em couve, obtiveram longevidades duas vezes maior que estas, com médias de 12 a 13 dias, respectivamente. 

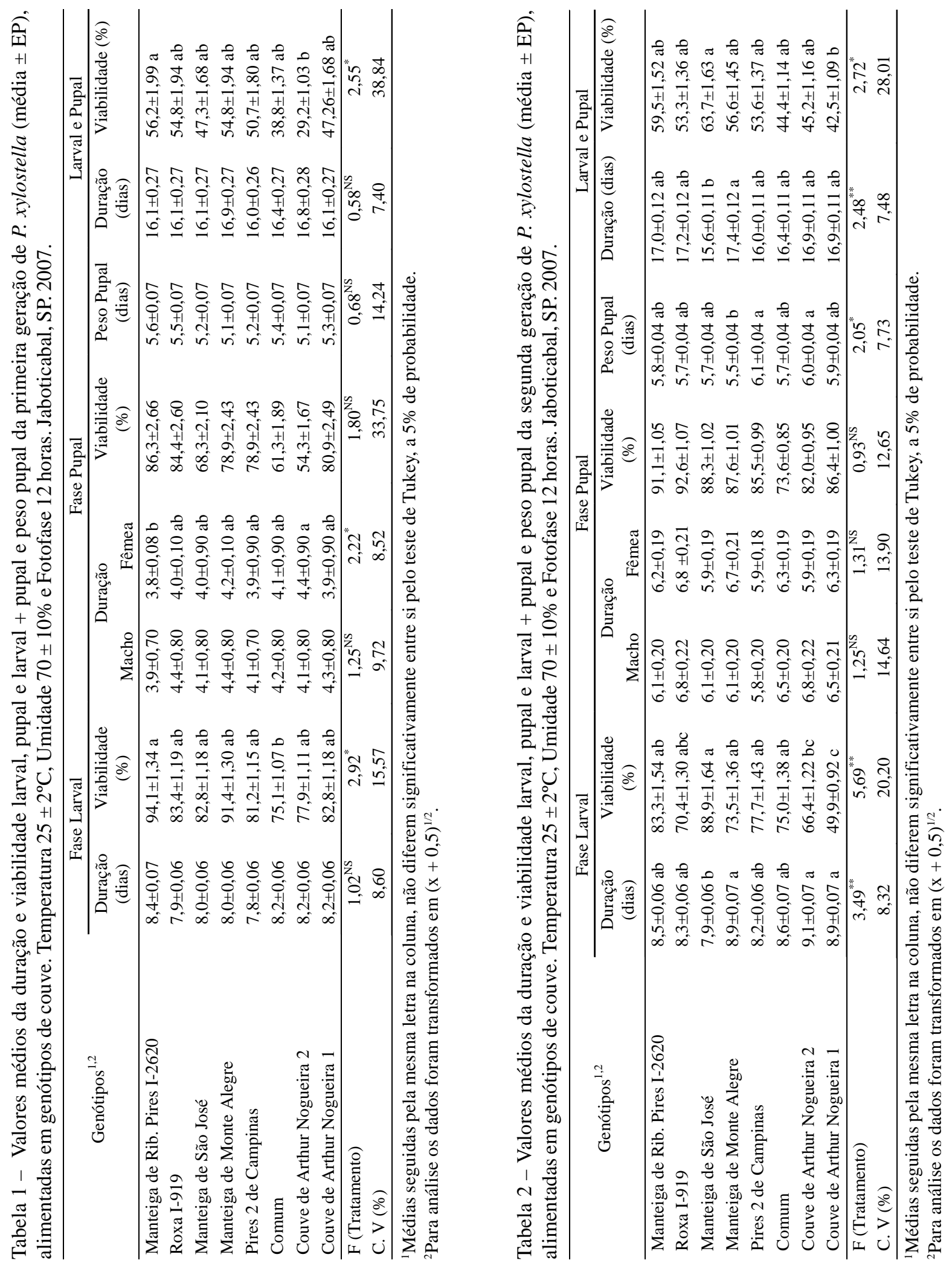


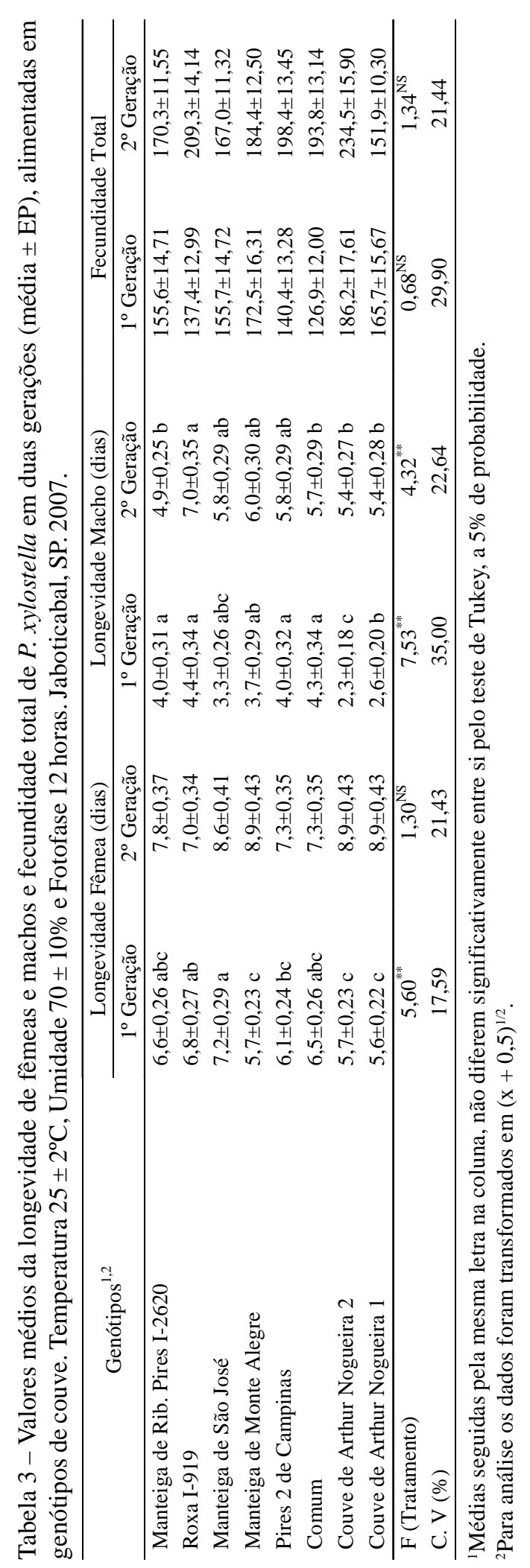

Ciênc. agrotec., Lavras, v. 35, n. 4, p. 710-717, jul./ago., 2011 
Os dados referentes ao peso de pupa, não alteraram consideravelmente entre os genótipos, resultados semelhantes foram obtidos por Ulmer et al. (2002), quando avaliou aspectos biológicos dessa praga em linhagens de mostarda, demonstrando a baixa interferência do hospedeiro para esse fator, quando avaliado em testes de resistência a $P$. xylostella.
Na segunda geração (Figura 2), a melhor separação foi em quatro grupos, utilizando a distância Euclidiana 5.

Apesar da mesma quantidade de agrupamentos, ocorreu diferenciações em relação à primeira geração, sendo o grupo 1 além de conter 'Couve de Arthur Nogueira 2' a inserção de Couve Comum, mostrando um efeito negativo

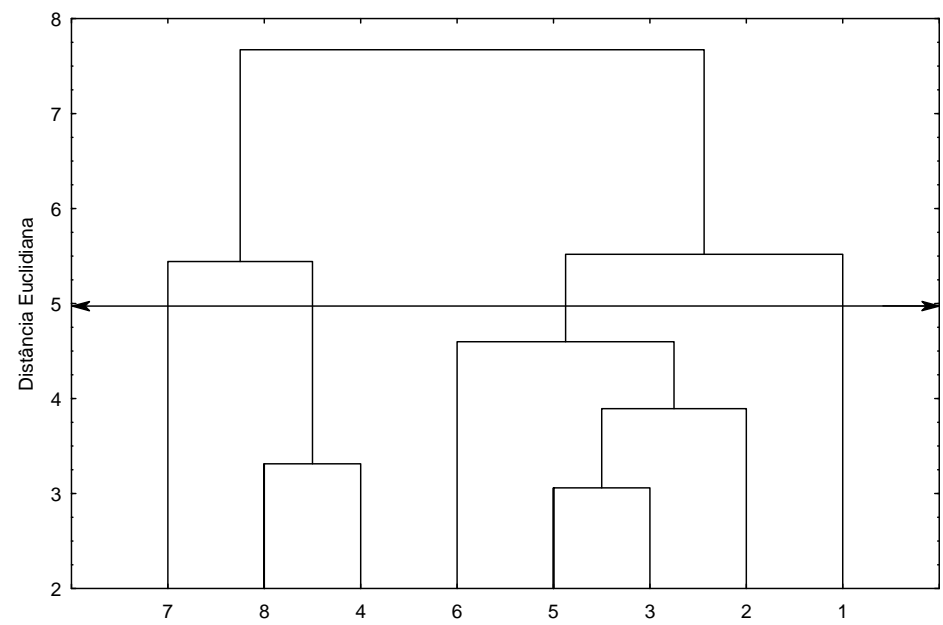

Figura 1 - Dendrograma baseado nos parâmetros biológicos de P. Xylostella alimentada em oito genótipos de couve na primeira geração. O método de aglomeração utilizado foi de Ward com a medida de distância de dissimilaridade euclidiana. Setas indicam a altura Euclidiana utilizada para separação dos grupos. 1: Manteiga de Ribeirão Pires I-2620, 2: Roxa I-919, 3: Manteiga de São José, 4: Manteiga de Monte Alegre, 5: Pires 2 de Campinas, 6: Comum, 7: Couve de Arthur Nogueira 2, 8: Couve de Arthur Nogueira 1.

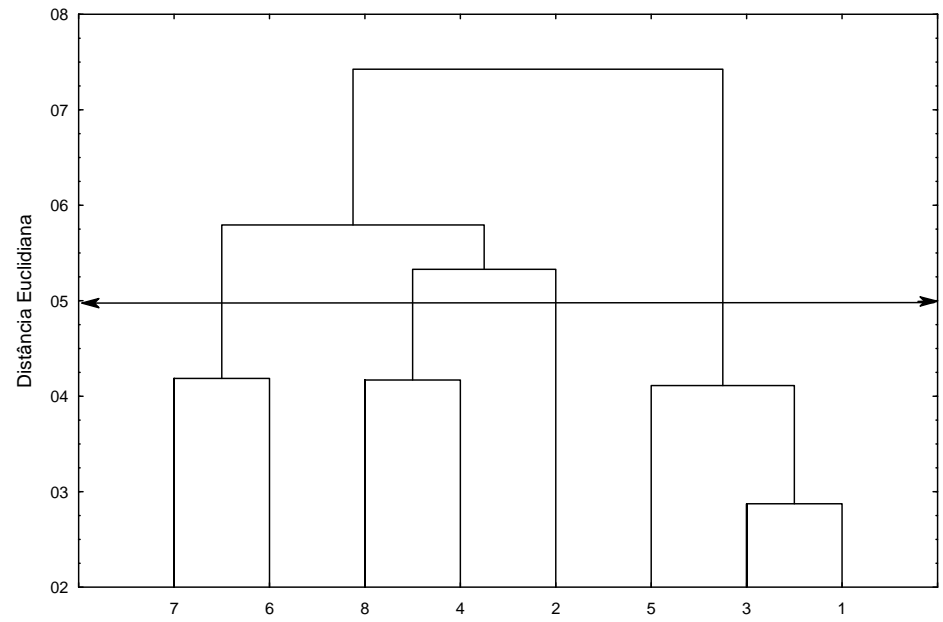

Figura 2 - Dendrograma baseado nos parâmetros biológicos de P. Xylostella alimentada em oito genótipos de couve na segunda geração. O método de aglomeração utilizado foi de Ward com a medida de distância de dissimilaridade euclidiana. Setas indicam a altura Euclidiana utilizada para separação dos grupos. 1: Manteiga de Ribeirão Pires I-2620, 2: Roxa I-919, 3: Manteiga de São José, 4: Manteiga de Monte Alegre, 5: Pires 2 de Campinas, 6: Comum, 7: Couve de Arthur Nogueira 2, 8: Couve de Arthur Nogueira 1 
mais significativo deste genótipo a $P$. xylostella quando sua segunda geração é criada sob o genótipo Comum novamente.

No grupo 3, dos quatro genótipos presentes na primeira geração, apenas 'Roxa I-919' permaneceu na segunda geração, estando 'Pires 2 de Campinas' e 'Manteiga de São José' no grupo 4, juntamente com 'Manteiga de Ribeirão Pires I-2620' que demonstrou-se estável a criação do inseto, sendo o genótipo mais suscetível durante as duas gerações avaliadas, podendo ser um material interessante para criação de $P$. xylostella em estudos de resistência de plantas a insetos.

\section{CONCLUSÕES}

Dentre os genótipos avaliados, Couve de Arthur Nogueira 2 é o menos favorável ao desenvolvimento de $P$. xylostella nas duas gerações, e Couve Comum demonstra maior influência negativa ao inseto na segunda geração.

O genótipo Manteiga de Ribeirão Pires I-2620 é o mais suscetível nas duas gerações, agrupando com este na segunda geração Pires 2 de Campinas e Manteiga de São José.

\section{AGRADECIMENTOS}

Ao Dr. Paulo Espíndola Trani, do Instituto Agronômico de Campinas (IAC) pelo fornecimento das mudas de couve para a instalação dos experimentos.

\section{REFERÊNCIAS BIBLIOGRÁFICAS}

BACCI, L. et al. Seletividade ecológica aos inimigos naturais de Plutella xylostella (L.) (Lepidoptera: Plutellidae) em brássicas. Ciência e Agrotecnologia, Lavras, v.33, p.2045-2051, 2009.

BARROS, R.; VENDRAMIM, J.D. Efeito de cultivares de repolho, utilizadas para criação de Plutella xylostella (L.) (Lepidoptera: Plutellidae), no desenvolvimento de Trichogramma pretiosum Riley (Hymenoptera: Trichogrammatidae). Anais da Sociedade Entomológica do Brasil, Londrina, v.28, n.3, p.469-476, 1999.

BOIÇA JUNIOR, A.L.; CHAGAS FILHO, N.R. Nãopreferência para alimentação de traça das crucíferas por genótipos de couve-flor. Arquivos do Instituto

Biológico, São Paulo, v.76, n.3, p.373-379, 2009.

EIGENBRODE, S.D.; SHELTON, A.M.; DICKSON, M.H. Two types of resistance to the diamondback moth (Lepidoptera: Plutellidae) in cabbage. Environmental Entomology, College Park, v.19, n.4, p.1086-1090, 1990.
EIGENBRODE, S.D. et al. Characteristics of glossy leaf waxes associated with resistance to diamondback moth (Lepidoptera: Plutellidae) in Brassica oleracea. Journal of Economic Entomology, Lanham, v.84, n.4, p.1609-1618, 1991.

GALLO, D. et al. Entomologia agrícola. Piracicaba: FEALQ, 2002.920p.

GONÇALVES, R.R.; DI MARE, R.A. Biologia da traça das crucíferas, Plutella xylostella Linnaeus (Lepidoptera, Yponomeutidae), sob condições controladas de temperatura, e parasitóides associados: part III, estudo sobre a biologia de Apanteles piceotrichosus (Blanchard) (Hymenoptera, Braconidae): análise do efeito de endocruzamento. Revista Brasileira de Zoologia, Curitiba, v.22, n.3, p.806-809, 2005.

HERNANDEZ, H.C.; VENDRAMIM, J.D. Avaliação da bioatividade de extratos aquosos de Meliaceae sobre Spodoptera frugiperda (J.E. Smith). Revista de Agricultura, Piracicaba, v.72, n.3, p.305-318, 1997.

\section{LARA, F.M. Princípios de resistência de plantas a} insetos. São Paulo: Ícone, 1991. 336p.

MARANHÃO, E.A. et al. Flutuação populacional da traça-das-crucíferas, em couve, na zona da Mata de Pernambuco. Horticultura Brasileira, Brasília, v.16, n.1, p.50-50, 1998.

NOGUEIRA, S.B. Pragas de brássicas. In: SIQUEIRA, T.S. (Ed.). Cultura de brássicas. Viçosa, MG: UFV, 1981. p.34-39.

SARFRAZ, M.L.; DOSDALL, L.M.; KEDDIE, B.A. Diamondback moth-host plant interactions: Implications for pest management. Crop Protection, Guildford, v.25, n.7, p.625-639, 2006.

SARFRAZ, M.L.; DOSDALL, L.M.; KEDDIE, B.A. Resistance of some cultivated Brassicaceae to infestations by Plutella xylostella (Lepidoptera: Plutellidae). Journal of Economic Entomology, Lanham, v.100, n.1, p.215-224, 2007.

SHELTON, A.M.; NAULT, B.A. Dead-end trap cropping: a technique to improve management of the diamondback moth, Plutella xylostella (Lepidoptera: Plutellidae). Crop Protection, Guildford, v.23, n.6, p.497-503, 2004. 
SPENCER, J.L.; PILLAI, S.; BERNAYS, E.A. Synergism in the oviposition behavior of Plutella xylostella: sinigrin and wax compounds. Journal of Insect Behavior, New York, v.12, n.4, p.483-500, 1999.

TAGLIARI, S.R.A. et al. Não-preferência alimentar de Plutella xylostella (L., 1758) (Lepidoptera: Plutellidae) por genótipos de Brassica oleracea L. var. acephala DC., em teste com chance de escolha. Arquivos do

Instituto Biológico, São Paulo, v.68, n.2, p.295-299, 2006.

THULER, R.T.; BORTOLI, A.S.; HOFFMANN-CAMPO, C.B. Classificação de cultivares de brássicas com relação à resistência à traça-das-crucíferas e à presença de glucosinolatos. Pesquisa Agropecuária Brasileira, Brasília, v.42, n.4, p.467-474, abr. 2007.

TORRES, A.L. et al. Efeito de extratos aquosos de Azadirachita indica, Melia azedarach e Aspidosperma pyrifolium no desenvolvimento e oviposição de Plutella xylostella. Bragantia, Campinas, v.65, n.3, p.447-457, 2006.

ULMER, B. et al. Diamondback moth, Plutella xylostella (L.), feeding and oviposition preferences on glossy and waxy Brassica rapa (L.). Crop Protection, Guildford, v.21, n.4, p.327-331, 2002. 